\title{
The Transition from Pre-Octonionic to Octonionic Gravity and How It May Be Pertinent to a Re-Do of the HUP for Metric Tensors
}

\author{
Andrew Walcott Beckwith \\ Physics Department, College of Physics, Chongqing University Huxi Campus, Chongqing, China \\ Email: Rwill9955b@gmail.com
}

How to cite this paper: Beckwith, A.W. (2017) The Transition from Pre-Octonionic to Octonionic Gravity and How It May Be Pertinent to a Re-Do of the HUP for Metric Tensors. Journal of High Energy Physics, Gravitation and Cosmology, 3, 727-753. https://doi.org/10.4236/jhepgc.2017.34055

Received: May 24, 2017

Accepted: October 24, 2017

Published: October 30, 2017

Copyright $\odot 2017$ by author and Scientific Research Publishing Inc. This work is licensed under the Creative Commons Attribution International License (CC BY 4.0).

http://creativecommons.org/licenses/by/4.0/ c) (i) Open Access

\begin{abstract}
The quantum gravity problem that the notion of a quantum state, representing the structure of space-time at some instant, and the notion of the evolution of the state, does not get traction, since there are no real "instants", is avoided by having initial Octonionic geometry embedded in a larger, nonlinear "pilot model" (semi classical) embedding structure. The Penrose suggestion of re-cycled space time avoiding a "big crunch" is picked as the embedding structure, so as to avoid the "instants" of time issue. Getting Octionic gravity as embedded in a larger, Pilot theory embedding structure may restore Quantum Gravity to its rightful place in early cosmology without the complication of then afterwards "Schrodinger equation" states of the universe, and the transformation of Octonionic gravity to existing space-time is explored via its possible linkage to a new version of the HUP involving metric tensors. We conclude with how specific properties of Octonion numbers algebra influence the structure and behavior of the early-cosmology model. This last point is raised in Section 14, and is akin to a phase transition from Pre-Octonionic geometry, in pre-Planckian space-time, to Octonionic geometry in Planckian space-time. A simple phase transition is alluded to; making this clear is as simple as realizing that Pre-Octonionic is for Pre-Planckian Space-time and Octonionic is for Planckian Space-time. We state that the Standard Model of physics occurs during Planckian Space-time. We also argue that the Standard Model does not apply to Pre Planckian Space-time. This is commensurate with the Octonion number system NOT applying in pre-Planckian spacetime, but applying in Plankian space-time. And the last line of Equation (54) gives a minimum time step in pre-Planckian space-time when we do NOT have the Standard Model of physics, or Octonionic Geometry.
\end{abstract}




\section{Keywords}

Octonionic Geometry, Cyclic Conformal Cosmology (Penrose), Modified HUP

\section{What Is Special about Octonionic Structure? Why Should One Care about It?}

Our plan is as follows. We state the Modified HUP results, as a Pre-Octonionic space-time result, and then we will specify that we are transitioning to Octonionic space time. The transition to Octonionic space time will then preserve one key result, that we have, due to the earlier pre-Octonionic space-time, a minimum time step.

In a word, this is the setup of the new physics, plus our resolution

1) In Pre-Octonionic (Pre-Planckian) Space-time there exist conditions for which we form an initial smallest time step, and that the Pre-Planckian Space-time is where we specify initially a modified HUP (Heisenberg Uncertainty principle).

2) In Octonionic (Planckian) Space-time, we recover QM and the usual HUP, but also, we have the benefits of keeping the minimum time step as to what is given from the Pre-Octonionic structure.

3) The Octonionic structure, as mentioned below, is $\mathrm{U}$ (1) cross $\mathrm{SU}$ (2) cross SU (3). In itself, the Octonionic structure only allows for the standard model physics, and so we will describe it below

4) To get "instants" of time, we need to go beyond the standard Model. After having said this, let us go to the construction of Octonionic non commutative geometry.

5) We will conclude with how specific properties of octonion numbers algebra influence the structure and behavior of the early-cosmology model. What one of the referees reviewing this document did not realize is that the Octonionic space time closely conforms to the Standard Model of physics, as has been stated repeatedly, and that the Pre-Octonionic state is when the Standard Model of physics does not apply. I.e. the division line between the Pre Octonionic Model and the Octonionic model directly correlates a transformation from Pre-Planckian physics to Planckian physics. This is thoroughly discussed in Section 14 of this manuscript.

Keep in mind one basic fact. If we restrict ourselves solely to Octonionic geometry, we are embedded deeply in only what the Standard Model of physics allows. We should though understand what is implied by the physics of the Octonionic structure and so the rest of this first discussion is devoted to it.

In [1] Wilson gives a generalized structure as to Octonionic geometry, and it is a generalized way to introduce higher level geometry into the formation of standard model physics. Crowell, in [2] examines its applications as to presumed 
space-time structure. Also note what is said in [3] the take away from it, is that as quoted from [3], that there exists

Quote:

(A linkage to the) mathematics of the division algebras and the Standard Model of quarks and leptons with $U(1) \times S U(2) \times S U(3)$ gauge fields

End of quote:

Once again, if we have only $U(1) \times S U(2) \times S U(3)$ gauge fields, we have only the standard model, and that if we wish to have a minimum time step, we need to go beyond the standard model.

The division algebras are linked to octonionic structure in a way which is touched upon by Crowell [2], but the main take away is that in the Pre-Planckian space-time regime, that there was specific non commutative structures, as reflected in the document below, which in Pre-Planckian space time would eventually become commutative. This development is illustrated in the text below.

The entire transition from Pre-Planckian space-time to Planckian space-time would be in tandem with findings by Beckwith, in [4], and [5] as to the physics, as given in both [4] [5] that kinetic energy would be greater than potential energy in the Pre-Planckian space-time regime, and also to the possibility of a causal discontinuity, as given in [6] which may be linked to the odd situation of which slow roll physics, as usually delineated by [7] becomes dominant,. It is also the considered opinion of the author that $E_{8}$ as referenced in [1] as well as [8] in a classical setting which may be linkable to the Octonionic structure, as well as an extension of issues brought up by Lisi in [9]. This is elaborated in greater detail in terms of Octonionic math in [10] by Baez.

Now that we have made note of the geometry, it is time to look at the metric tensor based fluctuations of space time which may be the bridge between the Pre-Planckian space-time behavior, and standard Planckian space-time

\section{The Basic Bridge, Looking at a Basic Re-Do of the HUP, in Terms of Metric Tensors, from [5]}

First of all, why would we have a different version of the HUP, in Pre-Octonionic geometry? So as to answer this question we will look at a Proto SUSY potential, and the inflaton, if $\phi \sim \xi^{+} \ll M_{\text {Planck }}$ which is what we assert we work with. This step, next then will allow us to reference an initial time step, which is non zero. We state that the HUP is modified, due to the existence of $\phi \sim \xi^{+} \ll M_{\text {Planck }}$ for an inflaton, and we outline what this deviance from the Standard model of physics says about the formation of an alternative statement of the HUP. From there we will then go to the use of the modified HUP to the formation of a minimum time step.

Now, start with the HUP as given in Pre-Planckian space-time Physics. As given in [5] we have that the following fluctuation may be germane to our problem, namely as given by a

Quote from [5] as 


\subsubsection{Examining What Happens to Equation (1) If in Pre-Planckian} Space Time $\dot{\phi}^{2} \gg V_{\text {Susy }}$ Due to $\phi \sim \xi^{+} \ll M_{\text {Planck }}$

If we look at the Susy potential as given by [11]

$$
V(\phi)=\mu^{4} \cdot\left[b \cdot \ln \left(\frac{\phi}{m_{\text {Planck }}}\right)+\left(1-\left(\frac{\phi}{m_{\text {Planck }}}\right)^{2}\right)^{2}\right]
$$

We will be looking at the value of Equation (1) if $\phi \sim \xi^{+} \ll M_{\text {Planck }}$. In short, we have then that

$$
\begin{aligned}
& (\Delta l)_{i j}=\frac{\delta g_{i j}}{g_{i j}} \cdot \frac{l}{2} \\
& (\Delta p)_{i j}=\Delta T_{i j} \cdot \delta t \cdot \Delta A \\
& \phi \sim \xi^{+} \ll M_{\text {Planck }}
\end{aligned}
$$

If we use the following, from the Roberson-Walker metric [11] [12] [13].

$$
\begin{aligned}
& g_{t t}=1 \\
& g_{r r}=\frac{-a^{2}(t)}{1-k \cdot r^{2}} \\
& g_{\theta \theta}=-a^{2}(t) \cdot r^{2} \\
& g_{\phi \phi}=-a^{2}(t) \cdot \sin ^{2} \theta \cdot d \phi^{2}
\end{aligned}
$$

Following Unruth [14] [15], write then, an uncertainty of metric tensor as, with the following inputs

$$
a^{2}(t)_{\min } \sim 10^{-110}, r \sim l_{P} \sim 10^{-35} \text { meters }
$$

Then, the surviving version of Equation (1) and Equation (2) is, then, if $\Delta T_{t t} \sim \Delta \rho$ [11] [14] [15]

$$
\begin{aligned}
& V^{(4)}=\delta t \cdot \Delta A \cdot r \\
& \delta g_{t t} \cdot \Delta T_{t t} \cdot \delta t \cdot \Delta A \cdot \frac{r}{2} \geq \frac{\hbar}{2} \\
& \Leftrightarrow \delta g_{t t} \cdot \Delta T_{t t} \geq \frac{\hbar}{V^{(4)}}
\end{aligned}
$$

This Equation (4) is such that we can extract, up to a point the HUP principle for uncertainty in time and energy, with one very large caveat added, namely if we use the fluid approximation of space-time [16]

$$
T_{i i}=\operatorname{diag}(\rho,-p,-p,-p)
$$

Then by [11]

$$
\Delta T_{t t} \sim \Delta \rho \sim \frac{\Delta E}{V^{(3)}}
$$

Then,

$$
\begin{aligned}
& \delta t \Delta E \geq \frac{\hbar}{\delta g_{t t}} \neq \frac{\hbar}{2} \\
& \text { Unless } \delta g_{t t} \sim O(1)
\end{aligned}
$$


This Change in the HUP, as outlined above, will be part and parcel of the transformation from Pre-Octonionic space time, to Octonionic, i.e. from PrePlanckian to Planckian physics, with all the resulting consequences, which will be outlined below

Before doing so, we say something about the introduction of what is meant by a metric tensor to begin with.

See the next mini session as to why the issue of the minimum fluctuation of the metric tensor is so important.

Having said this, we will be referring to Equation (7a) in our document as far as specifics, in the rest of this paper.

\subsubsection{Having Formed This Minimum HUP, as Given in Equation (7), Now How Do We Use It to Form a Minimum Time Step?}

The basic issue is, given as follows

$$
\begin{aligned}
& \delta t \Delta E \geq \hbar / \delta g_{t t} \\
& \Leftrightarrow \delta t g_{t t} \geq \frac{\hbar}{\delta g_{t t} \Delta E}
\end{aligned}
$$

The change in energy, as given in $\Delta E$ is enormous, i.e. almost equivalent to the entire energy budget of the Universe, at the start of the big bang, hence, to keep the minimum time step as larger than or equal to zero, it will require specific analysis of the fluctuation of the quantity $\delta g_{t t}$, but before doing this we need to understand what the metric tensor is physically, before initiating a description of what we are doing in Equation (7a) as to $\delta g_{t t}$.

\subsubsection{Introduction to the Metric Tensor as Contribution to Quantum Gravity: What Is Quantum Gravity? Does Quantum Gravity Have Relevance to Planckian Physics?}

In general relativity the metric $g_{a b}(\boldsymbol{x}, t)$ is a set of numbers associated with each point which gives the distance to neighboring points. I.e. general relativity is a classical theory. The problem is that in quantum mechanics physical variables, either as in (QED) electric and magnetic fields have uncertainty as to their values. As is well known if one makes an arbitrary, high accuracy position measurement of a quantum particle, one has lack of specific momentum values. I.e. its velocity. In Octonionic geometry, the commutation relationships are well defined. There is through a bridge between the classical regime of space time and its synthesis leading to a quantum result. It would be appropriate to put in specific constraints. Note that as an example in gauge theories, the idea is to use 'gauge fixing' to remove the extra degrees of freedom. The problem is though that in quantum theory, the resulting theory, (i.e. a quantum gravity theory) may not be independent of the choice of gauge. Secondly

In GR, it is possible to extract a time for each solution to the Einstein equations by DE parametrizing GR. Then the problem is, in quantum versions of cosmology that if space-time is quantized along these lines, the assumption (of evolving then quantizing) does not make sense in anything but an approximate way. That is, the resulting evolution does not generate a classical space-time! 
Rather, solutions will be wave-functions (solutions of some Schrödinger-type equation). What is being attempted HERE is to describe the limits of the quantum process so as to avoid having space time wave functions mandated to be Schrodinger clones. I.e. to restore quantum behavior as the geometric limit of specialized space time conditions.

Here is a problem. (In some approaches to canonical gravity, one fixes a time before quantizing, and quantizes the spatial portions of the metric only). Frankly fixing time before quantizing and then applying QM to just the spatial part is missing the point. If Quantum gravity is valid, then the commutation relationships in a definite geometric limit must hold. The paper refers to these regimes of space time where the octonionic commutation relations DO hold. The assertion made, is that before Planck temperature is reached, i.e. there is a natural embedding of space time geometry with the octonionic structure reached as the initial conditions for expansion of the present universe.

The premise followed in the paper is that before the Planckian regime, there are complex geometrical relationships involving quantum processes, but that the quantum processes are "hidden from view", due to their combination. The quantum processes are not measurable, in terms of specific quantum mechanical commutation relations until Planck temperature values (very high) are reached in terms of a buildup of temperature from an initially much lower temperature regime. Appendix A describes an embedding multi verse in terms of the present universe.

Rovelli [17] notes (2007, p. 1304), that modeling the gravitational field as an emergent, collective variable does not imply an absence of quantum effects, and it is possible that collective variables too are governed by quantum theory. Our re statement of this idea is to say that one has quantum effects emerging in highly specialized circumstances, with collective variables behaving like squeezed states of space time matter. The octonionic gravity regime, obeying quantum commutation behavior has its analog in simplification of collective variable treatment of a gravitational field, which becomes very quantum commutation like in its behavior in the Planck temperature limit. This paper will endeavor as to describe the emergent collective treatment of the gravitational field appropriately so octonionic gravity is a definite limiting structure emerging in extreme temperatures and state density.

\subsubsection{Conclusion as to What to Look forward to as Far as the Relevant} Transformation from Pre-Octonionic to Octonionic Structure

What we are considering is the following transformation, simply put. And this will be hopefully detected by a change in phase, given by phase $\delta_{0}$

$$
\begin{aligned}
& \delta t \Delta E \geq\left.\frac{\hbar}{\delta g_{t t}}\right|_{\text {Pre-Octonionic }} \stackrel{ }{\delta_{0} \text { phase change }} \delta t \Delta E \geq\left.\hbar\right|_{\text {Octonionic }} \\
& \text { with } \delta t \geq \frac{\hbar}{\delta g_{t t} \Delta E} \text { fixed }
\end{aligned}
$$




\section{Now about Conditions to Obtain the Relevant Data for Phase $\delta_{0}$}

This paper examines geometric changes that occurred in the earliest phase of the universe, leading to values for data collection of information for phase $\delta_{0}$, and explores how those geometric changes may be measured through gravitational wave data. The change in geometry is occurring when we have first a pre-quantum space time state, in which, in commutation relations [2] (Crowell, 2005) in the pre Octionic space time regime no approach to QM commutations is possible as seen by.

$$
\begin{aligned}
& {\left[x_{j}, p_{k}\right] \neq-\beta \cdot\left(l_{\text {Planck }} / l\right) \cdot \hbar \cdot T_{i j k}} \\
& \text { and does not } \rightarrow i \hbar \delta_{i, j}
\end{aligned}
$$

Equation (8) is such that even if one is in flat Euclidian space, and $i=j$, if there is no phase shift then there is no way to move beyond a flat space representation of

$$
\left[x_{j}, p_{k=j}\right] \neq\left. i \hbar\right|_{\text {Pre-Octonionic }}
$$

If one does not have the phase transition, then one observes that without the Pre-Octonionic to Octonionic phase shift that there is a permament stuck at the inequality given by Equation ( $8 \mathrm{a}$ ) above.

In the situation when we approach quantum "octonionic gravity applicable" geometry, Equation (8) becomes

$$
\left[x_{i}, p_{j}\right]=-\beta \cdot\left(l_{\text {Planck }} / l\right) \cdot \hbar \cdot T_{i j k} \underset{\text { approaching flat space after } \delta_{0}}{\longrightarrow} i \cdot \hbar \cdot \delta_{i, j}
$$

Equation (9) is such that even if one is in flat Euclidian space, and $i=j$, then if the phase transition from Pre-Octonionic to Octonionic has occurred,

$$
\left[x_{j}, p_{k=j}\right]=\left.i \hbar\right|_{\text {Octonionic }} \text { flat space Octonionic }
$$

.Also the phase change in gravitational wave data due to a change in the physics and geometry between regions where Equation (8) and Equation (9) hold will be given by a change in phase of GW, which may be measured inside a GW detector.

\section{Discussion of the Geometry Alteration Due to the Evolution from Pre-Planckian to Planckian Regimes of Space Time}

The simplest way to consider what may be involved in alterations of geometry is seen in the fact that in pre-octonionic space time regime (which is Pre-Planckian), one would have [2] (Crowell, 2005)

This Pre-Octonionic space-time behavior should be seen to be separate from the flatness condition as referred to in [18]. But retuning to [2] we have that, in Pre-Planckian space-time, that

$\left[x_{j}, x_{i}\right] \neq 0$ Under ANY circumstances, with low to high temperatures, or flat or curved space. (10) 
Whereas in the octonionic gravity space time regime where one would have Equation (9) hold that for enormous temperature increases (9), then by [2] (Crowell, 2005)

$$
\left[x_{j}, x_{i}\right]=i \Theta_{j, i} \underset{\mathrm{Temp} \rightarrow \infty}{\longrightarrow} 0
$$

Here,

$$
\Theta_{j, i} \sim \Lambda_{N C}^{-2} \sim \Lambda_{4-\text { dimensional }}^{-2} \propto 1 / T^{2 \beta} \underset{T \rightarrow \infty}{\longrightarrow} 0
$$

Specifically Equation (8) and Equation (10) will undergo physical geometry changes which will show up in $\delta_{0}$. The space time shift from pre Planck to the Planck epoch has gravity wave background radiation containing the imprint of the very earliest event. Next, is to consider what happens if Quantum (octonionic geometry) conditions hold. The supposition as given by in [19] (Lee, 2010)

Considering all these recent developments, it is plausible that quantum mechanics and gravity has information as a common ingredient, and information is the key to explain the strange connection between two.

When quantum geometry holds, as seen by Equation (9) and Equation (11), GW information is loaded into the octonion space time regime, and then transmitted to the present via relic GW which identified via the phase shift in GW as measured in a GW detector. This phase shift is $\delta_{0}$. The following flow chart is a bridge between the two regimes of [2] (Crowell, 2005) the case where the commutators for QM

$$
\begin{aligned}
& \underset{\left[x_{i}, p_{j}\right] \neq-\beta \cdot\left(l_{\text {Planck }} / l\right) \cdot \hbar \cdot T_{i j k}}{\stackrel{\text { Transition to Planckian space }}{\longrightarrow}\left[x_{i}, p_{j}\right]} \\
& =-\beta \cdot\left(l_{\text {Planck }} / l\right) \cdot \hbar \cdot T_{i j k}
\end{aligned}
$$

Equation (12) above represents the transition from pre-Planckian to Planckian geometry.

Also questions relating to how pre and post Planckian geometries evolve can be answered by a comparison of how entropy, in flat space geometry is linked with quantum mechanics [19] (Lee, 2010). Once Equation (12) happens, Beckwith hopes to look at the signals in phase shift $\delta_{0}$

$$
\underset{\left[x_{i}, p_{j}\right]=-\beta \cdot\left(l_{\text {Planck }} / l\right) \cdot \hbar \cdot T_{i j k}}{\stackrel{\text { Transition to release of relic gravitational waves in flat space }}{\text { Planckian era generated gravitational wave }}}
$$

Lee's paper [19] (Lee, 2010) gives the details of information theory transfer of information from initially curved space geometry to flat space. When one gets to flat space, then, by Equation (13) one then has a release of relic GW. The readers are referred to appendix A summarizing the relevant aspects of [19] (Lee, 2010) in connecting space time geometry (initially curved space, of low initial degrees of freedom) to Rindler geometry for the flat space regime occurring when degrees of freedom approach a maxima, initially from $t>0 \mathrm{~s}$ up to about $t<1 \mathrm{~s}$ as outlined in an argument given in Equation (14). One of the primary results is 
reconciling the difference in degrees of freedom versus a discussion of dimensions. Also, as Equation (12) occurs, there will be a buildup in the number of degrees of freedom, from a very low initial level to a higher one, as in the Gaussian mapping [20] (Beckwith, 2010)

$$
x_{i+1}=\exp \left[-\tilde{\alpha} \cdot x_{i}\right]+\tilde{\beta}
$$

The feed in of temperature from a low level, to a higher level is in the prePlanckian to Planckian thermal energy input as by (Beckwith, 2010a) [21]

$$
E_{\text {thermal }} \approx \frac{k_{B}}{2} \cdot T_{\text {temperature }} \propto \Omega_{0} \cdot T_{\text {temperature }} \sim \tilde{\beta}
$$

Equation (14) would have low numbers of degrees of freedom, with an eventual Gauss mapping up to 100 to 1000 degrees of freedom, as described by (Kolb and Turner, 1990) [21]. The rest of this paper will be in describing an extension of an idea by [22] (Beckwith, 2011c) which may give multiple universes as put into Equation (15). And [22] about multiple universes uses [23] explicitly, by Penrose. In reality, what we are doing is equivalent to [24], which has the useful caveat that

Quote

We propose that in time dependent backgrounds the holographic principle should be replaced by the generalized second law of thermodynamics.

End of quote.

As there have been numerous ways to add in an active time component into Pre-Planckian space time physics, to Planckian, this substitution of a generalized $2^{\text {nd }}$ law of thermodynamics is equivalent to the transformation from Pre Plankian to Planckian space-time, which again is in a $1-1$ correlation to when we are doing which is to go to Pre-Octonionic to Octonionic structures, and we will be elaborating upon this point in the next several parts of this manuscript. Starting off with the sequential development of $\mathrm{VeV}$ (Vacuum expectation values) and emergent space-time physics.

\section{Details of the Model, in Terms of the VeVs Used for Space Time Evolution. How to Set up Cosmological Inputs into Our Universe to Get Appropriate Values of $\delta_{0}$}

Further elaboration is tied in with a summary of properties of a mutually unbiased basis (MUB), [25] (Chaturvedi, 2007) which is topologically adjusted to properties of flat space Rindler geometry.

$\delta_{0}$. The key point is an inter relationship between a change in MUB, from initial highly complex flat space time, as a new way to quantify a phase transition, for experimentally verifiable detection of $\delta_{0}$. The values of $\delta_{0}$ are set by the difference between Renyi entropy [26] Salvail, 2009), and a particle count version of entropy, i.e. $S \sim\langle n\rangle$. The topological transition is due to a change in basis/geometry from the regime of Renyi entropy to entropy in a particle count 
version of entropy, i.e. $S \sim\langle n\rangle$ [27] (Ng, 2008). As by [28] (Beckwith and Glinka. 2010) (assuming a vacuum energy $\rho_{\text {Vacuum }}=[\Lambda / 8 \pi \cdot G]$ initially), with $\Lambda$ part of a closed FRW Friedman Equation solution.

$$
a(t)=\frac{1}{\sqrt{\Lambda / 3}} \cosh [\sqrt{\Lambda / 3} \cdot t]
$$

To flat space FRW equation of the form (Beckwith and Glinka, 2010) [28]

$$
(\dot{a}(t) / a(t))^{2}+(1 / a(t))^{2}=\Lambda / 3
$$

Beckwith tried inputs into the initial value of $\Lambda$ as high energy fluctuations, this $\rho_{\text {vaccum }}=\Lambda / 8 \pi G$ links initial vacuum expectation value $(\mathrm{VeV})$ behavior with the following diagram. Note that cosmology models have to be consistent with the following Figure 1.

As stated by [30] (Crowell, 2010), the way to delineate the evolution of the $\mathrm{VeV}$ is to consider an initially huge $\mathrm{VeV}$, due to inflationary geometry. Note by Equation (18), [31] (Poplawski, 2011):

$$
\rho_{\Lambda}=H \cdot \lambda_{\mathrm{QCD}}
$$

Where $\lambda_{\mathrm{QCD}}$ is $200 \mathrm{MeV}$ and similar to the QCD scale parameter of the SU (3) gauge coupling constant, where $H$ a Hubble parameter. Here if there is a relationship between Equation (18) above and $\rho_{\text {vacum }}=\Lambda / 8 \pi G$ then the formation of inputs into our vacuum expectation values $V \sim V_{\mathrm{VEV}} \sim 3\langle H\rangle^{4} / 16 \pi^{2}$, and equating $V \sim V_{\mathrm{VEV}} \sim 3\langle H\rangle^{4} / 16 \pi^{2}$ with $V(\phi) \sim \phi^{2} / 2$ would be consistent with an inflaton treatment of inflation which has similarities to [32] (Kuchiev and $\mathrm{Yu}$, 2008). Then equate vacuum potential with vacuum expectation values as:

$$
\begin{aligned}
& \rho_{\text {vaccum }}=\Lambda / 8 \pi G=\rho_{\Lambda}=H \cdot \lambda_{\mathrm{QCD}} \\
& \Leftrightarrow V \sim V_{\mathrm{VEV}} \sim 3\langle H\rangle^{4} / 16 \pi^{2} \approx V(\phi) \sim \phi^{2}
\end{aligned}
$$

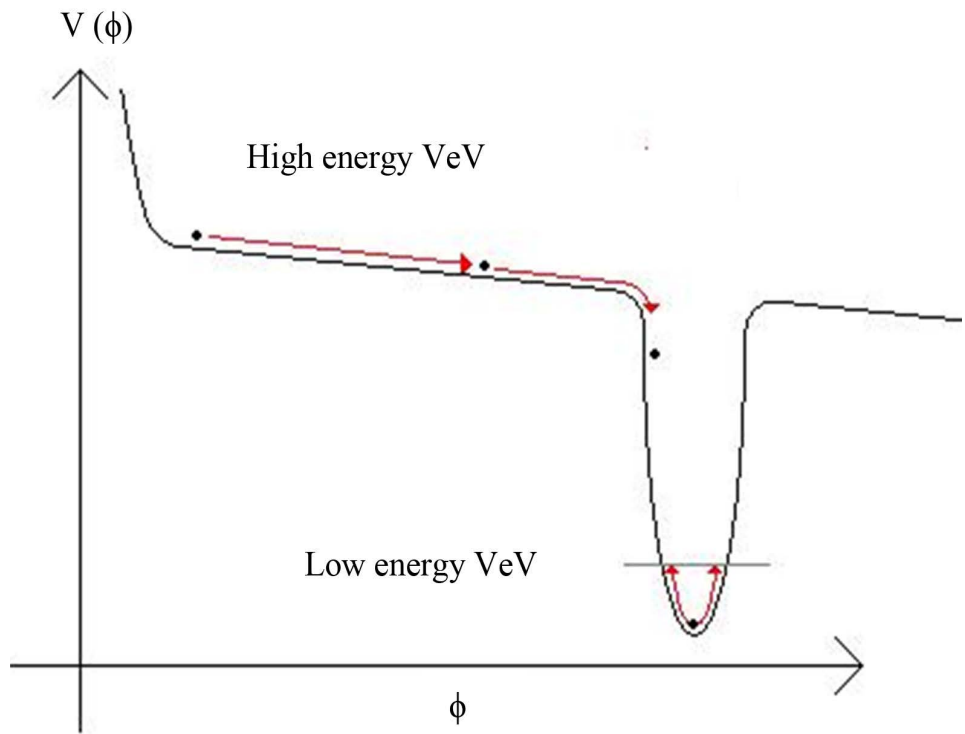

Figure 1. As supplied by (Crowell, 2010) [29]. 
Different models for the Hubble parameter, $H$ exist, and are linked to how one forms the inflaton. The author presently explore what happens to the relations as given in Equation (14) before, during, and after inflation. Table 1 below. Is how to obtain inflation?

\section{First, Thermal Input into the New Universe. In Terms of Vacuum Energy}

We will briefly allude to temperature drivers which may say something about how thermal energy will be introduced into the onset of a universe. Begin first with [33] (Beckwith, 2008)

$$
\left|\Lambda_{5-\operatorname{dim}}\right| \approx c_{1} / T^{\alpha}
$$

In contrast with the traditional four-dimensional version of the same, as given by [34] (Park, 2002)

$$
\Lambda_{4-\operatorname{dim}} \approx c_{2} \cdot T^{\beta}
$$

If one looks at the range of allowed upper bounds of the cosmological constant, the difference between what [35] (Barvinsky, 2006) predicted, and [34] (Park, 2002) is:

$$
\begin{aligned}
& \Lambda_{4-\text { dim }} \approx c_{2} \cdot T^{\beta} \underset{\text { Graviton production for time } t>t \text { (Planck) }}{\longrightarrow} 360 \cdot m_{\text {Planck }}^{2} \\
& \ll c_{2} \cdot\left(T \approx 10^{32} \text { Kelvin }\right)^{\beta}
\end{aligned}
$$

Right after the gravitons are released, one sees a drop-off of temperature contributions to the cosmological constant. Then for time values $t \approx \delta^{1} \cdot t_{\text {Planck }} ; 0<\delta^{1} \leq 1$ and integer $\mathrm{n}$ [33] (Beckwith, 2008)

$$
\frac{\Lambda_{4-\operatorname{dim}}}{\left|\Lambda_{5-\operatorname{dim}}\right|}-1 \approx n^{-1}
$$

Initial phases of the big bang, with large vacuum energy $\neq \infty$ and $a\left(t_{\text {min }}\right) \neq 0, a\left(t_{\text {min }}\right) \ll 1$ then

Table 1 may suggest a discontinuity in the pre-Planckian regime, for scale factors [35] (Beckwith, 2008).

$$
\left[\frac{a\left(t^{*}+\delta t\right)}{a\left(t^{*}\right)}\right]-1<(\text { value }) \approx \varepsilon^{+} \ll 1
$$

Table 1. Cosmological $\Lambda$ in 5 and 4 dimensions (Beckwith, 2008) [33].

\begin{tabular}{ccc}
\hline $\begin{array}{c}\text { Time } \\
0 \leq t \ll t_{\text {Planck }}\end{array}$ & Time $0 \leq t \leq t_{\text {Planck }}$ & Time $t \geq t_{\text {Planck }}$ \\
\hline$\Lambda_{5} \mid$ undefined, & $\left|\Lambda_{5}\right| \approx \varepsilon^{+}$, & \\
$T \approx \varepsilon^{+}$Kelvin & $T \approx 10^{32}$ Kelvin $>T$ & $\frac{\Lambda_{4-\text { dim }}}{\left|\Lambda_{5-\text { dim }}\right|}-1 \approx 0^{+}$Temperature \\
$\rightarrow T \approx 10^{32}$ Kelvin & $>T \approx 10^{12}$ Kelvin \\
$\Lambda_{4-\text { dim }} \sim \infty$ & $\Lambda_{4-\text { dim }} \sim$ Huge & much lower.
\end{tabular}


Furthermore, the assumption is that there is an initial fixed entropy, with $\bar{N}$ as a nucleated structure in short time interval as temperature

$T_{\text {temperature }} \in\left(0^{+}, 10^{19} \mathrm{GeV}\right)$ arrives. Then [36] (Beckwith, 2010a)

$$
\begin{aligned}
& S=\frac{\hbar}{T} \cdot\left[2 k-\frac{\tilde{\tilde{B}}}{\eta^{2}}\right]^{1 / 2} \sim n_{\text {particle count }} \\
& \& \tilde{\tilde{B}}=m_{\text {Planck }}^{2} \cdot\left(\left(-\frac{3}{\pi}\right) \phi^{-2}\right)
\end{aligned}
$$

If the inputs into the inflaton $\phi$, as given by a random influx of thermal energy from temperature, we will see the particle count on the right hand side of Equation (25) as a random creation of $n_{\text {particle count }}$. The way to introduce the expansion of the degrees of freedom from zero to $N(T) \sim 10^{2}-10^{3}$ is to define the classical and quantum regimes of gravity to minimize the point of the bifurcation diagram affected by quantum processes As by [20] [36] (Beckwith, 2010) defined $\frac{\Delta \tilde{\beta}}{\text { dist }} \sim$ Change in degrees of freedom

$$
\frac{\Delta \tilde{\beta}}{\text { dist }} \cong 5 k_{B} \Delta T_{\text {temperature }} \sim q \cdot E_{\text {net electric field }}
$$

Equation (20) is the regime in which we see a thermal increase in temperature, up to the Planckian regime. If so, then we can next look at what is the feeding in mechanism from the end of a universe, or universes, and inputs into Equation

\section{A New Idea Extending Penrose's Suggestion of Cyclic Universes, Black Hole Evaporation, and the Embedding Structure Our Universe Is Contained within}

Beckwith strongly suspects that there are no fewer than $N$ universes undergoing Penrose 'infinite expansion' [20] [23] (Penrose, 2006) contained in a mega universe structure. Furthermore, each of the $N$ universes has black hole evaporation, with the Hawking radiation from decaying black holes. If each of the $N$ universes is defined by a partition function, called $\left\{\Xi_{i}\right\}_{i=N}^{i=1}$, then there exist an in formation ensemble of mixed minimum information correlated as about $10^{7}$. $10^{8}$ bits of information per partition function in the set $\left.\left\{\Xi_{i}\right\}_{i \equiv N}^{i=1}\right|_{\text {before }}$, so minimum information is conserved between a set of partition functions per universe

$$
\left.\left.\left\{\Xi_{i}\right\}_{i=N}^{i=1}\right|_{\text {before }} \equiv\left\{\Xi_{i}\right\}_{i=N}^{i=1}\right|_{\text {after }}
$$

However, there is non-uniqueness of information put into each partition function $\left\{\Xi_{i}\right\}_{i \equiv N}^{i=1}$. Furthermore Hawking radiation from the black holes is collated via a strange attractor collection in the mega universe structure to form a new big bang for each of the $N$ universes represented by $\left\{\Xi_{i}\right\}_{i \equiv N}^{i=1}$. Verification of this mega structure compression and expansion of information with a nonuniqueness of information placed in each of the $N$ universes favors Ergodic 
mixing treatments of initial values for each of $N$ universes expanding from a singularity beginning. The $n_{f}$ value, will be using [27] ( $\left.\mathrm{Ng}, 2008\right)$

$S_{\text {entropy }} \sim n_{f}$. How to tie in this energy expression, as in Equation (28) (23) will be to look at the formation of a non-trivial gravitational measure as a new big bang for each of the $\mathrm{N}$ universes as by $n\left(E_{i}\right)$. the density of states at a given energy $E_{i}$ for a partition function. [31]

$$
\left\{\Xi_{i}\right\}_{i=1}^{i \equiv N} \propto\left\{\int_{0}^{\infty} \mathrm{d} E_{i} \cdot n\left(E_{i}\right) \cdot \mathrm{e}^{-E_{i}}\right\}_{i \equiv 1}^{i \equiv N} .
$$

Each of $E_{i}$ identified with Equation (28) above are with the iteration for $N$ universes (Penrose, 2006)

$$
\begin{aligned}
& \left.\frac{1}{N} \cdot \sum_{j=1}^{N} \Xi_{j}\right|_{j \text { before nucleation regime }} \\
& \text { vacuum nucleation tranfer } \\
& \left.\Xi_{i}\right|_{i \text { fixed after nucleation regime }}
\end{aligned}
$$

For $N$ number of universes, with each $\left.\Xi_{j}\right|_{j \text { before nucleation regime }}$ for $j=1$ to $N$ being the partition function of each universe just before the blend into the RHS of Equation (29) above for our present universe. Also, each of the independent universes given by $\left.\Xi_{j}\right|_{j \text { before nucleation regime }}$ are constructed by the absorption of one million black holes taking in energy. I.e., [22] [23] (Penrose, 2006)

$$
\left.\left.\Xi_{j}\right|_{j \text { before nucleation regime }} \approx \sum_{k=1}^{\max } \tilde{\Xi}_{k}\right|_{\text {black holes jth universe }}
$$

\section{Analysis of the Action of These Two Mappings on the Formation of Quantum Gravity}

In particular, in the regime where there is a buildup of temperature, [2] Crowell, 2005) Equation (31) gives

$$
\begin{aligned}
& \oint\left[x_{i}, p_{j}\right] \cdot \mathrm{d} x_{k} \\
& =-p_{i} \oint\left[x_{j}, \mathrm{~d} x_{k}\right] \\
& =-\beta \cdot l_{\text {Plank }} \cdot T_{j k i} \oint p_{i} \cdot \mathrm{d} x_{j} \\
& \neq-\hbar \cdot \beta \cdot l_{\text {Plank }} \cdot T_{i j k}
\end{aligned}
$$

Very likely, across a causal boundary, between $\pm l_{P}$ across the boundary due to the causal barrier, one gets [2] (Crowell, 2005)

$$
\oint p_{i} \cdot \mathrm{d} x_{k} \neq \hbar \cdot \delta_{i k} \oint p_{i} \cdot \mathrm{d} x_{k}=0
$$

I.e.

$$
\left.\oint_{ \pm l_{\text {Plank }}} p_{i} \cdot \mathrm{d} x_{k}\right|_{i=k} \rightarrow 0
$$

If so, [2] (Crowell, 2005) the regime of space time, for the feed in of, prior to the introduction of QM, that Equation (33) in itself would mean that in the prePlanckian physics regime, and in between $\pm l_{p}$, QM no longer applies. 


\section{Formal Proof That Increase in Thermal Temperatures as Given in Table 1 Leads to Approaching Quantum Mechanics}

We look at the [37] (Ecker,2007) article as to how to look at the way one may have, if temperatures increase, as stated in Table 1 above, from a low point to a higher one, for a flattening of space time. This noncommutative geometry due to rising temperatures signifies conditions for the emergence of Equation (4) to become [2] (Crowell, 2005)

$$
\left[x_{j}, p_{i}\right] \underset{\mathrm{Temp} \rightarrow \infty}{\longrightarrow} i \hbar \delta_{i, j}
$$

In order to get conditions for Equation (34) we referred to non-commutative geometry breakdown [37] (Ecker, 2007)

$$
\Theta_{j i} \sim i \Lambda_{N C}^{-2} \sim i \cdot\left[\Lambda_{4-\mathrm{Dim}}\right]^{-2} \propto i /\left[T^{2 \beta}\right] \underset{T \rightarrow \infty}{\longrightarrow} 0
$$

When Equation (35) goes to zero we submit that Equation (35) is recovering quantum/Octoinian gravity. Equation (35) above, according to the [37] (Ecker, 2007), page 79, is linkable to initial violations of Lorentz invariance. The claim is that the entire argument of Equation (35) with rising temperature is a way to understand the removal of non-Euclidian space to approach Euclidian flat space. Beckwith shall next examine how this increasing temperature may lead to an explosion of the degrees of freedom present.

\section{Understanding How Phase Shift in Gravitational Waves May Be Affected by the Transition to a Causal Discontinuity, and Different Models of Emergent Structure}

In research work as given by [38] (Li, and Yang, 2009), [39] (Beckwith, 2010b) outlined in Chongqing November 2010 the following representation of amplitude, i.e. as by reading [38] (Li, and Yang, 2009) the following case for amplitude

$$
A_{\otimes}=A_{\oplus}=\breve{A}
$$

Furthermore, first order perturbative terms of an E\&M field have its components written as. (Li, and Yang, 2009)

$$
\tilde{F}_{0,2}^{(1)}=\tilde{F}_{0,1}^{(1)}
$$

Secondly, there is a way to represent the" number" of transverse first order perturbative photon flux density as given in an earth bound high frequency GW detector [38]. (Li, and Yang, 2009).

$$
\begin{gathered}
n_{r}^{(1)}=\frac{c}{2 \mu_{0} \hbar \omega_{e^{-}}} \cdot \operatorname{Re}\{\} \\
\{\}=i \cdot[\exp [-i \theta]] \cdot \tilde{F}_{0,1}^{(1) *}\left[\frac{i}{\omega_{e^{-}}} \cdot\left(\frac{\partial \Psi_{x}}{\partial y}-\frac{\partial \Psi_{y}}{\partial x}\right)\right]
\end{gathered}
$$

Here the quantity $\frac{i}{\omega_{e^{-}}} \cdot\left(\frac{\partial \Psi_{x}}{\partial y}-\frac{\partial \Psi_{y}}{\partial x}\right)$ represents the $z$ component of the 
magnetic field of a Gaussian beam used in an EM cavity to detect GW. We introduce the quantity $Q$, the quality factor of the detector cavity set up to observe $\mathrm{GW}$, and $\breve{A}$, the experimental GW amplitude. In the simplest case, $B_{y}^{(0)}$ is a static magnetic field. Then $\tilde{F}_{0,1}^{(2)}=i \cdot \tilde{F}_{0,1}^{(1)}$ leads to [38] (Li, and Yang, 2009)

$$
\tilde{F}_{0,1}^{(1)}=i \cdot 2 \breve{A} \cdot B_{y}^{(0)} \cdot Q \cdot\left(\sin \left[\frac{n \pi z}{b}\right]\right) \cdot \exp \left\{i \cdot\left(-\omega_{g} t+\delta_{0}\right)\right\}
$$

The formula $E_{\text {thermal }} \approx \frac{k_{B}}{2} \cdot T_{\text {temperature }} \propto \Omega_{0} \cdot T_{\text {temperature }} \sim \tilde{\beta}$ from Equation(15) is a feed into $\omega_{g}$ provided time $t \propto$ Planck time, and set Equation (40) with $\omega_{g} \sim \omega_{e^{-}}$by setting up $E_{\text {thermal }} \approx \frac{k_{B}}{2} \cdot T_{\text {temperature }} \sim \tilde{\beta}$. In other words, for relic GW production, an interrelationship between $\tilde{\alpha}$ and $E_{\text {thermal }} \approx \frac{k_{B}}{2} \cdot T_{\text {temperature }} \sim \tilde{\beta}$ for increases in degrees of freedom. This is a different perspective than what is normally used in analyzing what happens in a transition between initial Planck time $\sim 10^{-44}$ seconds, and cosmological evolution up to $10^{-30}$ seconds The next discussion is on research done by [38] (Li, et al., 2003), as to identifying traces of massive gravitons. [39] (Beckwith, 2011b)

\section{Re Casting the Problem of GW/Graviton in a Detector for "Massive" Gravitons}

We now turn to the problem of detection. The following discussion is based upon with the work of $\mathrm{Li}$, Beckwith, and other physics researchers in Chongqing University.(Li, et al., 2003), [40] (Beckwith,2010b) [39]. What (Li et al., 2003) [40] have shown in 2003 which Beckwith made an extension (Beckwith, 2011b) [41] is to obtain a way to present first order perturbative electromagnetic power flux, i.e. $T^{u v}$ in terms of a non-zero four dimensional graviton rest mass, in a detector, in the presence of uniform magnetic field (Li et al., 2003) [40], (Beckwith, 2010b) [41]. What if we have curved space time with an energy momentum tensor of the electromagnetic fields in GW fields as given by (Li et al., 2003) [40]?

$$
T^{u v}=\frac{1}{\mu_{0}} \cdot\left[-F_{\alpha}^{\mu} F^{v \alpha}+\frac{1}{4} \cdot g^{\mu v} F_{\alpha \beta} F^{\alpha \beta}\right]
$$

(Li et al., 2003) [40] States that $\tilde{F}_{u v}=\tilde{F}_{u v}^{(0)}+\tilde{F}_{u v}^{(1)}$, with $\left|\tilde{F}_{u v}^{(1)}\right| \ll\left|\tilde{F}_{u v}^{(0)}\right|$ will lead to

$$
T^{u v}=\stackrel{(0)}{(0)} \stackrel{(1)}{u v} \stackrel{(2)}{(2)}
$$

The $1^{\text {st }}$ term to the right side of Equation (42) is the energy-momentum tensor of the back ground electromagnetic field, and the $2^{\text {nd }}$ term to the right-hand side of Equation (42) is the first order perturbation of an electromagnetic field due to the presence of gravitational waves [39].

$$
J_{\text {eff }} \cong n_{\text {count }} \cdot m_{4-\text { dim-(Graviton) }}
$$


Table 2. Managing GW generation from Pre-Planckian physics [43] (Maggiorie, 2000), [44] (Beckwith, 2011).

\begin{tabular}{lll}
\hline$h_{C} \leq 2.82 \times 10^{-33}$ & $f_{\mathrm{GW}} \sim 10^{12}$ Hertz & $\lambda_{\mathrm{GW}} \sim 10^{-4}$ meters \\
$h_{C} \leq 2.82 \times 10^{-29}$ & $f_{\mathrm{GW}} \sim 10^{8}$ Hertz & $\lambda_{\mathrm{GW}} \sim 10^{0}$ meters \\
$h_{C} \leq 2.82 \times 10^{-25}$ & $f_{\mathrm{GW}} \sim 10^{4}$ Hertz & $\lambda_{\mathrm{GW}} \sim 10^{1}$ kilometers \\
$h_{C} \leq 2.82 \times 10^{-23}$ & $f_{\mathrm{GW}} \sim 10^{2}$ Hertz & $\lambda_{\mathrm{GW}} \sim 10^{3}$ kilometers \\
\hline
\end{tabular}

As stated [42] $m_{4 \text {-dim-(Graviton) }} \sim 10^{-65}$ grams, while $n_{\text {count }}$ is the number of gravitons which may be in the detector sample. What Beckwith and Li intend to do is to isolate out an $T^{u v}$ assuming $_{1)}$ a non-zero graviton rest mass and $\mu_{(1)}$ $\tilde{\beta}=|F|$ and make a linkage with $T^{00}$. The term $T^{00}$ isolated out from $T^{u v}$. The point is that detected GW helps constrain Equation (43). If this is done, the next step will be different GW measurement protocols. As one can try working with, using the ideas of [43] we can set

$$
h_{0}^{2} \Omega_{\mathrm{GW}} \sim 10^{-6}
$$

Next we note the results of using $h_{0}^{2} \Omega_{\mathrm{GW}} \sim 10^{-6}$ in GW measurements

\section{Wavelength, Sensitivity and Other Such Constructions from Maggiore, with Our Adaptations and Comments}

We will next give several basic considerations as to early universe geometry which are appropriate as to the [43] (Maggiore, 2000) treatment of both wavelength, strain, and $\Omega_{\mathrm{GW}}$. The idea will be to look at how the ten to the tenth stretch out of generated wave length may tie in with early universe models. We want to, if $h_{0}=0.51 \pm 0.14$, understand what affects an expansion of GW wave lengths.

What Beckwith expects, [2] Crowell, 2011) is that initial waves, in the Planckian regime have about $\lambda_{\mathrm{GW}} \sim 10^{-14}$ meters for $f_{\mathrm{GW}} \sim 10^{22}$ Hertz which would turn into $\lambda_{\mathrm{GW}} \sim 10^{-1}$ meters for $f_{\mathrm{GW}} \sim 10^{9} \mathrm{Hertz}$, and sensitivity of $h_{C} \leq 2.82 \times 10^{-30}$. It is important to note that the $h_{0}^{2} \Omega_{\mathrm{GW}} \sim 10^{-6}$ is the first measurement metric which is drastically altered $h_{C}$. Which is mentioned in Equation (44c) is an upper bound. In reality, only the $2^{\text {nd }}$ and $3^{\text {rd }}$ columns in Table 1 above escape being inaccurate., since the interactions of gravitational waves/gravitons with quark-gluon plasmas deform by an order of magnitude $h_{C}$. So for Table 1, the first column is an upper bound which, even if using Equation (44c) is off by an order of magnitude. More seriously, the number of gravitons per unit volume of phase space is dependent upon $h_{0}^{2} \Omega_{\mathrm{GW}} \sim 10^{-6}$. If that is changed, Equation (44b) is less valid. Beckwith refers the readers to [44] (Beckwith, 2011c) which gives Equation (40b) values.

The particle per phase state count is, [43] (Maggiorie, 2000)

$$
n_{f} \sim h_{0}^{2} \Omega_{\mathrm{GW}} \cdot \frac{10^{37}}{3.6} \cdot\left[\frac{1000 \mathrm{~Hz}}{f}\right]^{4}
$$


Secondly detector strain for device physics is given by [43] (Maggiorie, 2000)

$$
h_{C} \leq\left(2.82 \times 10^{-21}\right) \cdot\left(\frac{1 \mathrm{~Hz}}{f}\right)
$$

These values of strain, the numerical count, and $n_{f}$ give a bit count and entropy which will lead to limits as to how much information is transferred. Note after the start of inflation with at the beginning of relic inflation

$\lambda_{\mathrm{GW}} \sim 10^{-1}$ meters $\Rightarrow n_{f} \propto 10^{6}$ graviton/unit phase space for $f_{\mathrm{GW}} \sim 10^{9} \mathrm{Hertz}$ This is to have, say a starting point in pre inflationary physics of $f_{\mathrm{GW}} \sim 10^{22} \mathrm{Hertz}$ when $\lambda_{\mathrm{GW}} \sim 10^{-14}$ meters, i.e. a change of $\sim 10^{13}$ orders of magnitude in about $10^{-25}$ seconds. The challenge will be to come up with an input model which will justify a new data model, [43] (Maggiorie, 2000)

\section{Providing a Curve for the Fifth Cosmology Model, as a Modification/Extension of the Penrose Model}

One can look now at the following approximate model for the discontinuity put in, due to the heating up implied in Table 1. This is [33] (Beckwith, 2008)

$$
\frac{\Lambda_{\max } V^{(4)}}{8 \pi G} \sim T^{00} \cdot V^{(4)} \sim \rho \cdot V^{(4)}=E_{\text {Total }}
$$

The approximation in this treatment is that $E_{\text {total }} \propto V(\varphi)$ where we are looking at a potential energy term. [35] (Barvinsky, 2006). What we pay attention to, is an exponential potential [45] (Weinberg, 2008)

$$
V(\varphi)=g \cdot \varphi^{\alpha}
$$

What we come up with pre, and post Planckian space time regimes, when looking at consistency of the emergent structure is the following. Namely, [45] (Weinberg, 2008),

$$
\begin{gathered}
\qquad(\phi) \propto \varphi^{|\alpha|} \text { for } t<t_{\text {PLanck }} \\
\text { Also, we would have } V(\phi) \propto 1 / \varphi^{|\alpha|} \text { for } t \gg t_{\text {PLanck }}
\end{gathered}
$$

The switch between Equation (47a) and Equation (47b) is not provable. And the author designates this as the boundary of a causal discontinuity. According to [45] (Weinberg, 2008), if $\in=\frac{\lambda^{2}}{16 \pi G}, H=1 / \in t$ so that a scale factor be-haves as

$$
a(t) \propto t^{1 / \epsilon}
$$

Then, if (Weinberg, 2008) [46]

$$
|V(\varphi)| \ll(4 \pi G)^{-2}
$$

There are no quantum gravity effects. I.e., if one uses an exponential potential a scalar field could take the value of, when there is a drop from $\phi_{1}$ to $\phi_{2}$ for flat space geometry [45] (Weinberg, 2008) 


$$
\varphi(t)=\frac{1}{\lambda} \ln \left[\frac{8 \pi G g \in^{2} t^{2}}{3}\right]
$$

Then the scale factors, from [45] (Weinberg, 2008)

$$
\frac{a\left(t_{2}\right)}{a\left(t_{1}\right)}=\left(\frac{t_{2}}{t_{1}}\right)^{1 / \epsilon}=\exp \left[\frac{\left(\varphi_{2}-\varphi_{1}\right) \lambda}{2 \epsilon}\right]
$$

The more $\frac{a\left(t_{2}\right)}{a\left(t_{1}\right)} \gg 1$, then the less likely there is a tie in with quantum gravity.

Note those that the way this potential is defined is for a flat, Roberson-Walker geometry, and that if $t_{1}<t_{\text {Planck }}$ then Equation (51) no longer applies, and that one is not having connection with an Octonionic Gravity regime.

\section{We Are Then Going to Get the Following Expression for the Energy/Frequency Spread in the Penrose Alternation of the Big "Crunch" Model}

Start with working with the expression given by Equation (15). This is for time $\breve{T} \sim 0^{+}$to $10^{-44}$ seconds, $\Omega_{\mathrm{GW}} \sim 10^{6}$ and a frequency variance

$$
\Omega_{0} \in[1 \mathrm{GHz}, 10 \mathrm{GHz}]
$$

This Equation (52) is due to $T_{\text {temperature }} \sim 10^{32}$ Kelvin at the point of generation of the discontinuity leading to a discontinuity for a signal generation as given by $\delta_{0}$ at $\breve{T} \sim 10^{-44}$ seconds. This process above. is for inputs into $\Omega_{0} \breve{T} \sim \tilde{\beta}$ The assumption is that the discontinuity, as given by $\delta_{0}$ getting to temperature $T_{\text {temperature }} \sim 10^{32}$ Kelvin, for $\Omega_{\mathrm{GW}} \sim 10^{6}$, meaning that the peak curve of frequency will be between 1 to $10 \mathrm{GHz}$ for $\Omega_{\mathrm{GW}} \sim 10^{6}$, with a falling value of $\Omega_{\mathrm{GW}}$ for frequencies $<1 \mathrm{GHz}$

(1)

\section{Can a Researcher Find an Appropriate $T^{u v}$ If One Has Nonzero Graviton Rest Mass?}

It depends upon understanding what is meant by emergent structure to generalize what is known in mathematics as the concept of "self-organized criticality. In 2001, [46] (Zimmermann and Voelcker, 2001) refer to an abstract mathematical self-organized criticality structure. We assert that the mathematical self-organized criticality structure is akin to a definition as to how Dp branes arise at the start of inflation. What is the emergent structure permitting $\oint p_{i} \cdot \mathrm{d} x_{k}=\hbar \cdot \delta_{i k}$ to hold? What is the self-organized criticality structure leading to forming an appropriate $T^{(1)}$ if one has non-zero graviton rest mass? Answering such questions will permit us to understand how to link $T^{u v}$ in a GW detector, to $\tilde{\beta}$ in Equation (8). The following construction is used to elucidate how an EM Gaus sian beam can be used to help in isolating $T^{u v}$ in a GW detector. One of the main things to consider is resolution of the following: [47] (Feeney, et al. 2011) at University College London say they've found evidence of four collisions 
with other universes in the form of circular patterns in the cosmic microwave background. In their model, called "eternal inflation," the universe is a bubble in a much larger cosmos. This cosmos is filled with other bubbles, all of which are other universes where the laws of physics may be different from ours. As seen in Figure 2. This also echoes [49] (Smolin, 1997).

Chongqing university researchers are attempting to add more information than Figure 2 above, via suitable analysis of $T^{(1)}$, [48] (Gurzadyan, Penrose, 2011).

\section{Presenting How Specific Properties of Octonion} Numbers Algebra Influence the Structure and Behavior of the Early-Cosmology Model. And Why the Bridge from Pre-Planckian to Planckian Physics Is So Important to This Situation

As has been stated, repeatedly, Pre-Planckian Space-time physics is also the realm of the Pre-Octonionic geometry. Planckian Space-time physics is when Octonionic geometry holds. Once again the point was made in the beginning of this manuscript and it will be repeated again for emphasis

Quote:

(A linkage to the) mathematics of the division algebras and the Standard Model of quarks and leptons with $U(1) \times S U(2) \times S U(3)$ gauge fields

End of quote:

Once again, if we have only $U(1) \times S U(2) \times S U(3)$ gauge fields, we have only the standard model, and that if we wish to have a minimum time step, we need to go beyond the standard model. We can only go to beyond the Standard Model in the Pre Octionion geometry. And we will not have a minimum time step, in the $U(1) \times S U(2) \times S U(3)$ situation.

The main point. $U(1) \times S U(2) \times S U(3)$ occurs in Octonionic geometry, and that Planckian Space time regime is where we lock in the Standard Model in Planckian Space-time physics. This is commensurate with [1] [2], and [3], whereas we do not have this $U$ (1) $\times S U(2) \times S U$ (3) in Pre-Octonionic geometry.

Furthermore, we can bring up the minimum time step in the

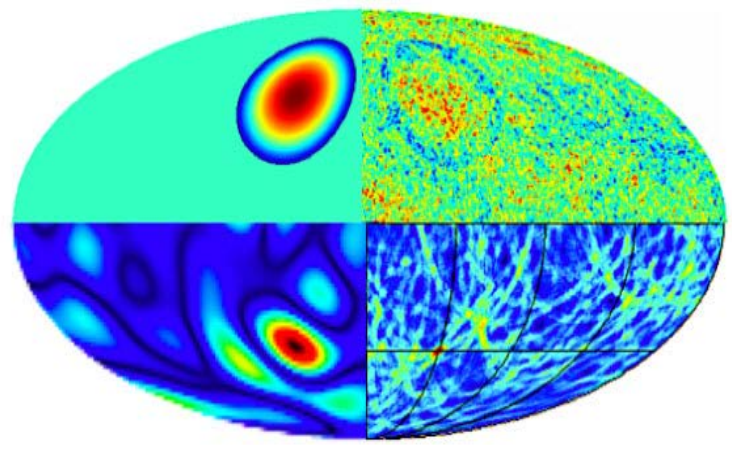

Figure 2. Based upon: First Observational Tests of Eternal Inflation [47] (Feeney, et al. 2011). 


\section{Pre-Octonionic geometry, which corresponds to Pre-Planckian Space- time physics.}

The minimum time step, as has been iterated again and again is commensurate due to the physics of the Pre-Octonionic regime of space time. I.e. we get a minimum time step as given by looking at [50] when we can come up a different Heisenberg uncertainty principle in the Pre-Planckian space-time, which is also brought up explicitly in [18] as to read,

$$
\begin{aligned}
& V^{(4)}=\delta t \cdot \Delta A \cdot r \\
& \delta g_{t t} \cdot \Delta T_{t t} \cdot \delta t \cdot \Delta A \cdot \frac{r}{2} \geq \frac{\hbar}{2} \\
& \Leftrightarrow \delta g_{t t} \cdot \Delta T_{t t} \geq \frac{\hbar}{V^{(4)}}
\end{aligned}
$$

This further is simplified in [50] to read to the Pre-Planckian HUP relationship leading to a minimum time step of

$$
\begin{aligned}
& V^{(4)}=\delta t \cdot \Delta A \cdot r \\
& \delta g_{t t} \cdot \Delta T_{t t} \cdot \delta t \cdot \Delta A \cdot \frac{r}{2} \geq \frac{\hbar}{2} \\
& \Leftrightarrow \delta g_{t t} \cdot \Delta T_{t t} \geq \frac{\hbar}{V^{(4)}} \\
& \Rightarrow \Delta E \Delta t \geq \frac{\hbar}{\delta g_{t t}},\left|\delta g_{t t}\right| \ll 1 \\
& \Rightarrow \Delta t \geq \frac{\hbar}{\Delta E \cdot \delta g_{t t}}
\end{aligned}
$$

Notice that in the Pre-Planckian space time, we need to have a fluctuation of energy $\Delta E$, and there is in the last line no requirement for clearly delineated space-time. We further state this last line becomes in minimum uncertainty an equality leading then in the Pre-Planckian space-time conditions for a minimum value of the time step. This is why we even invoked Octonionic geometry, and its break down in the Pre-Planckian Space-time.

\section{Conclusion: In Terms of the Planckian Evolution, as Well as the Contribution into It from Different Universes}

Analog, reality feed in from other universes may be the driving force behind the evolution of inflationary physics. We presume going to Octonionic gravity is then, quantum [51] (Beckwith, 2011c). Pre-Octonionic gravity physics (analog regime of reality) features a breakdown of the Octonionic gravity commutation relationships when one has curved space time. This corresponds, as brought up in the Jacobi iterated mapping for the evolution of degrees of freedom to a buildup of temperature for an increase in degrees of freedom from 2 to over 100 per unit volume of space time. The peak regime of where the degrees of freedom maximize is where the Octonionic regime holds. Analog physics, prior to the buildup of temperature can be represented by Equation (1) and Equation (3). The input into Equation (1) and Equation (3) is Equation (24) which is an er- 
godic mapping, from many universes into our own present universe. This mapping requires a deterministic quantum limit as similar to what [52] ( $t^{\prime} H o o f t$, 2006). Theoretically, inputs into Equation (1) and Equation (3) await experimentally falsifiable experiments. If what the author suspects, i.e. ergodic characteristics may be leading to a feed into Equation (1) and Equation (3) due to Equation (24). We want verification of Equation (24).

Note that [24] has the following quote

Quote

A modified form of the holographic bound that applies to a post-inflationary universe follows from the generalized second law. However, in a spatially closed universe, or inside a black hole event horizon, there is no simple relationship that connects the area of a region to the maximum entropy it can contain.

The choice between these two reflects upon if there is a multiverse, or if there is, even more to the point if there is information transfer and mixing between components of universes which may hold if the following quasi ergodic process holds, according to [53].

We also have to be aware of the startling possibility raised in [54], namely that Quote

In theories in which the cosmological constant takes a variety of values in different "subuniverses," the probability distribution of its observed values is conditioned by the requirement that there be someone to measure it. This probability is proportional to the fraction of matter that is destined to condense out of the background into mass concentrations large enough to form observers. We calculate this "collapsed fraction" with a simple, pressure-free, spherically symmetric, nonlinear model for the growth of density fluctuations in a flat universe with arbitrary value of the cosmological constant, applied in a statistical way to the observed spectrum of density fluctuations at recombination.

I.e. our model of the evolution of a cosmological constant, as given in the use of the Park model, as in [34] we are considering possible "subuniverse" contributions to the cosmological constant, as given by modeling the cosmological constant as conflated with Dark Energy. As given in [22].

However, there is another way to model a "cosmological constant" as given in [55], namely a model for the cosmological "constant" which is a consequence of the generalized HUP they derive. Their HUP though has none of the flourishes put in, as far as the work which has been alluded to for Pre-Planckian to Planckian physics evolution as reported in [4], [5] and as in the beginning of our text.

I.e. a worthy project would be to differentiate between either choosing [54] or [55] and if a variant of [50] is chosen, to substitute the HUP as given by [50] by what has been derived and published in [4].

The details of such a choice would have profound implications as far as heavy gravity, as well as the current given in Equation (43). In addition, the details of the Pre-Planckian to Planckian Space-time could be investigated more thoroughly. 
We argue that the details of the division algebras, and the links to Octonionic geometry as alluded to in the beginning of the text would be either falsified, by experimental measurements, or confirmed, which could lead to researchers adding more details as to [1] [2] and [3] as well as confirming the central importance of what Lisi attempted working with in [9]. It would be interesting and perhaps useful to compare this with the predictions given by Abbot [56] a s well as the revising the issue which is brought up by reference [57]. Keep in mind as well that there has been recent confirmation by Abbot [58] as to the existence of gravitational waves, which further extends what was brought up by Abbot, et al. of the LIGO observational team, which is in terms of black hole binaries, which further confirms the solutions of the issues, brought up by Abbot in [56] as well as also room to explore the insights brought up by Corda in [59] which await further investigation.

\section{Acknowledgements}

This work is supported in part by National Nature Science Foundation of China grant No. 11375279

\section{References}

[1] Wilson, R. (2008) Octonions. http://www.maths.qmul.ac.uk/ raw/talks_files/octonions.pdf

[2] Crowell, L. (2005) Quantum Fluctuations of Spacetime World Press Scientific. World Scientific Series in Contemporary Chemical Physics, Vol. 25, Singapore.

[3] Dixon, G.M. (1994) Division Algebras: Octonions Quaternions Complex Numbers and the Algebraic Design of Physics. Mathematics and Its Applications, Kluwer Academic Publishers, London. https://doi.org/10.1007/978-1-4757-2315-1

[4] Beckwith, A. (2016) Gedanken Experiment Examining How Kinetic Energy Would Dominate Potential Energy, in Pre-Planckian Space-Time Physics, and Allow Us to Avoid the BICEP 2 Mistake. Journal of High Energy Physics, Gravitation and Cosmology, 2, 75-82. https://doi.org/10.4236/jhepgc.2016.21008

[5] https://www.researchgate.net/publication/296077757_Gedankenexperiment_for_ini ti-

al_expansion_of_the_universe_and_effects_of_a_nearly_zero_inflaton_in_Pre_Plan ckian_physics_space-time_satisfying_traditional_Slow_Roll_formulas_which_happ ens_in_Pre_Planck

[6] Beckwith, A. (2016) Could a Causal Discontinuity Explain Fluctuations in the CMBR Radiation Spectrum? http://vixra.org/abs/1602.0035

[7] Padmanabhan, T. (2005) Understanding Our Universe, Current Status and Open Issues. In: Ashatekar, A., Ed., 100 Years of Relativity, Space-Time Structure: Einstein and Beyond, World Scientific Publishing, Singapore, 175-204. http://arxiv.org/abs/gr-qc/0503107

[8] Lee, S. (2007) The Plebanski Action Extended to a Unification of Gravity and Yang-Mills Theory.

[9] Lisi, A.G. (2011) Garrett Lisi Responds to Criticism of His Proposed Unified Theory of Physics. Scientific American.

[10] Baez, J. (2002) The Octonians. Bulletin of the American Mathematical Society, 39, 
145-205. http://arxiv.org/abs/math/0105155v4

[11] Kolb, E., Pi, S. and Raby, S. (1984) Phase Transitions in Super Symmetric Grand Unified Models. In: Fang, L. and Ruffini, R., Eds., Cosmology in the Early Universe, World Press Scientific, Singapore, 45-70.

[12] Beckwith, A. (2016) Gedanken Experiment for Refining the Unruh Metric Tensor Uncertainty Principle via Schwartz Shield Geometry and Planckian Space-Time with Initial Nonzero Entropy and Applying the Riemannian-Penrose Inequality and Initial Kinetic Energy for a Lower Bound to Graviton Mass (Massive Gravity). Journal of High Energy Physics, Gravitation and Cosmology, 2, 106-124. https://doi.org/10.4236/jhepgc.2016.21012

[13] Gorbunov, D. and Rubakov, V. (2011) Introduction to the Theory of the Early Universe, Cosmological Perturbations and Inflationary Theory. World Scientific Publishing, Singapore.

[14] Unruh, W.G. (1986) Why Study Quantum Theory? Canadian Journal of Physics, 64, 128-130. https://doi.org/10.1139/p86-019

[15] Unruh, W.G. (1986) Erratum: Why Study Quantum Theory? Canadian Journal of Physics, 64, 128-130. https://doi.org/10.1139/p86-019

[16] Giovannini, M. (2008) A Primer on the Physics of the Cosmic Microwave Background. World Press Scientific, Hackensack. https://doi.org/10.1142/6730

[17] Rovelli, C. (2008) Complete LQG Propagator. II. Asymptotic Behavior of the Vertex. Physical Review D, 77, Article ID: 044024. https://doi.org/10.1103/PhysRevD.77.044024

[18] Beckwith, A. (2016) Gedanken Experiment for Degree of Flatness, or Lack of, in Early Universe Conditions. Journal of High Energy Physics, Gravitation and Cosmology, 2, 57-65. https://doi.org/10.4236/jhepgc.2016.21006

[19] Lee, J.W. (2010) Quantum Mechanics Emerges from Information Theory Applied to Causal Horizons. Found Phys, 1-10. https://doi.org/10.1007/s10701-010-9514-3

[20] Beckwith, A. (2010) How to Use the Cosmological Schwinger Principle for Energy Flux, Entropy, and "Atoms of Space-Time" to Create a Thermodynamic Space-Time and Multiverse. http://iopscience.iop.org/article/10.1088/1742-6596/306/1/012064

[21] Kolb, E. and Turner, S. The Early Universe. Westview Press, Chicago.

[22] Beckwith, A. (2014) Analyzing Black Hole Super-Radiance Emission of Particles/Energy from a Black Hole as a Gedankenexperiment to Get Bounds on the Mass of a Graviton. Advances in High Energy Physics, 2014, Article ID: 230713. http://www.hindawi.com/journals/ahep/2014/230713/

[23] Penrose, R. (2006) Before the Big Bang. An Outrageous New Perspective and Its Implications for Particle Physics. Proceedings of EPAC, Edinburgh, 2759-2763.

[24] Easther, R. and Lowe, D.A. (1999) Holography, Cosmology and the Second Law of Thermodynamics. Physical Review Letters, 82, 4967-4970.

http://arxiv.org/abs/hep-th/9902088 https://doi.org/10.1103/PhysRevLett.82.4967

[25] Chaturvedi, S. (2007) Mutually Unbiased Bases. Pramana Journal of Physics, 52, 345-350.

[26] Revzen, M. and Khanna, F.C. Encryption via Entangled States Belonging to Mutually Unbiased Bases. https://arxiv.org/pdf/0809.1945.pdf

[27] Ng, Y.J. (2008) Spacetime Foam: From Entropy and Holography to Infinite Statistics and Nonlocality. Entropy, 10, 441-461. https://doi.org/10.3390/e10040441 
[28] Beckwith, A.W. and Glinka, L. (2010) The Arrow of Time Problem: Answering if Time Flow Initially Favouritizes One Direction Blatantly. Prespacetime Journal, 1, 1358-1375. http://vixra.org/abs/1010.0015

[29] Crowell, L. (2010) In Private Communication to A.W. Beckwith.

[30] Crowell, L. (2011) In Private Communication with Author.

[31] Poplawski, N. (2011) Cosmological Constant from QCD Vacuum and Torsion. Annalen der Physik, 523, 291. http://arxiv.org/abs/1005.0893v1

[32] Kuchiev, M.Y. (1998) Can Gravity Appear Due to Polarization of Instantons in SO(4) Gauge Theory? Classical and Quantum Gravity, 15, 1895-1913.

[33] Beckwith, A.W. (2008) AIP Conference Proceedings, 969, 1018-1026.

[34] Park, D.K., Kim, H. and Tamarayan, S. (2002) Nonvanishing Cosmological Constant of Flat Universe in Brane world Senarios. Physics Letters B, 535, 5-10.

[35] Barvinsky, A., Kamenschick, A. and Yu, A. (2006) Thermodynamics from Nothing: Limiting the Cosmological Constant Landscape. Physical Review D, 74, Article ID: 121502.

[36] Beckwith, A.W. (2010) Applications of Euclidian Snyder Geometry to the Foundations of Space-Time Physics. EJTP, 7, 241-266.

[37] Ecker, G. Effective Field Theories. In: Pierre, J., Francoise, G.L. and Naber, T.S., Eds., Encyclopedia of Mathematical Physics, Vol. 4, Gauge Theory, Quantum Field Theory, Sheung Tsun, 77-85.

[38] Li, F. and Yang, N. (2009) Phase and Polarization State of High Frequency Gravitational Waves. Chinese Physics Letters, 236, Article ID: 050402.

[39] Beckwith, A.W. (2010) Presentation in Chongqing University.

[40] Li, F., Tang, M. and Shi, D. (2003) Electromagnetic Response of a Gaussian Beam to High Frequency Relic Gravitational Waves in Quintessential Inflationary Models. Physical Review D, 67, Article ID: 104008. https://doi.org/10.1103/PhysRevD.67.104008

[41] Goldhaber, A. and Nieto, M. (2010) Photon and Graviton Mass Limits. Reviews of Modern Physics, 82, 939-979. http://arxiv.org/abs/0809.1003 https://doi.org/10.1103/RevModPhys.82.939

[42] Giovannini, M. (1999) Production and Detection of Relic Gravitons in Quintessential Inflationary Models. Physical Review D, 60, Article ID: 123511.

http://arxiv.org/abs/astro-ph/9903004 https://doi.org/10.1103/PhysRevD.60.123511

[43] Maggiore, M. (2000) Gravitational Wave Experiments and Early Universe Cosmology. Physics Reports, 331, 283-367.

[44] Beckwith, A.W. Detailing Minimum Parameters as Far as Red Shift, Frequency, Strain, and Wavelength of Gravity Waves/Gravitons, and Possible Impact upon GW Astronomy. http://vixra.org/pdf/1103.0020v1.pdf

[45] Weinberg, S. (2008) Cosmology. Oxford University Press.

[46] Zimmermann, R. (1997) Topoi of Emergence Foundations \& Applications. http://arxiv.org/pdf/nlin/0105064 v1

[47] Feeney, S.M., et al. (2011) First Observational Tests of Eternal Inflation. http://arxiv.org/abs/1012.1995

[48] Gurzadyan, V.G. and Penrose, R. (2010) Concentric Circles in WMAP Data May Provide Evidence of Violent Pre-Big-Bang Activity. http://arxiv.org/abs/1011.3706

[49] Smolin, L. (1997) The Life of the Cosmos. Oxford University Press, Oxford. 
[50] Beckwith, A.W. (2016) Addition to the Article with Stepan Moskaliuk on the Inter Relationship of General Relativity and (Quantum) Geometrodynamics, via Use of Metric Uncertainty Principle. Journal of High Energy Physics, Gravitation and Cosmology, 2, 467-471

[51] Beckwith, A.W. Do Physical Laws/ Physics Parameter Constants Remain Invariant from a Prior Universe, to the Present Universe? http://vixra.org/abs/1708.0474

[52] Hooft, G. (2006) Beyond the Quantum. World Press Scientific, Singapore. http://arxiv.org/PS_cache/quant-ph/pdf/0604/0604008v2.pdf

[53] Dye, H.A. (1965) On the Ergodic Mixing Theorem. Transactions of the American Mathematical Society, 118, 123-130. https://doi.org/10.1090/S0002-9947-1965-0174705-8

[54] Martel, H., Shapiro, P. and Weinberg, S. (1998) Likely Values of the Cosmological Constant. The Astrophysical Journal, 492, 29-40. http://iopscience.iop.org/article/10.1086/305016/fulltext/35789.text.html

[55] Miao, Y.-G. and Zhao, Y.-J. (2014) Interpretation of the Cosmological Constant Problem within the Framework of Generalized Uncertainty Principle. International Journal of Modern Physics D, 23, Article ID: 1450062.

http://arxiv.org/abs/1312.4118 https://doi.org/10.1142/S021827181450062X

[56] Abbott, B.P., et al. (2009) An Upper Limit on the Stochastic Gravitational-Wave Background of Cosmological Origin, Data. Nature, 460, 990.

http://www.phys.ufl.edu/ tanner/PDFS/Abbott09Nature-Stochastic.pdf https://doi.org/10.1038/nature08278

[57] Clarkson, C. and Seahra, S. (2007) A Gravitational Wave Window on Extra Dimensions. Classical and Quantum Gravity, 24, F33. https://doi.org/10.1088/0264-9381/24/9/F01

[58] Abbot, B.P., et al. (2016) Observation of Gravitational Waves from a Binary Black Hole Merger. Physical Review Letters, 116, Article ID: 061102. https://physics.aps.org/featured-article-pdf/10.1103/PhysRevLett.116.061102

[59] Corda, C. (2009) Interferometric Detection of Gravitational Waves: The Definitive Test for General Relativity. International Journal of Modern Physics D, 18, 2275-2282. https://arxiv.org/abs/0905.2502 https://doi.org/10.1142/S0218271809015904 


\section{Appendix A: Highlights of J.-W. Lee's Paper [19]}

The following formulation is to highlight how entropy generation blends in with quantum mechanics, and how the breakdown of some of the assumptions used in Lee's paper coincide with the growth of degrees of freedom. What is crucial to Lee's formulation, is Rindler geometry, not the curved space formulation of initial universe conditions. First of all. [19] (Lee, 2010),

"Considering all these recent developments, it is plausible that quantum mechanics and gravity has information as a common ingredient, and information is the key to explain the strange connection between two. If gravity and Newton mechanics can be derived by considering information at Rindler horizons, it is natural to think quantum mechanics might have a similar origin. In this paper, along this line, it is suggested that quantum field theory (QFT) and quantum mechanics can be obtained from information theory applied to causal (Rindler) horizons, and that quantum randomness arises from information blocking by the horizons

To start we look at the Rindler partition function, as by [19] (Lee, 2010)

$$
\begin{aligned}
Z_{R} & =\sum_{i=1}^{n} \exp \left[-\beta H\left(x_{i}\right)\right] \\
& =\operatorname{Trace}(\exp [-\beta H])
\end{aligned}
$$

As stated by Lee [48] we set $Z_{R}$ to be equal to the quantum mechanical partition function of a particle with mass $m$ in Minkowski space-time. Furthermore, there exists the datum that: Lee made an equivalence between Equation (A1) and [19] (Lee, 2010)

$$
Z_{Q}=N_{1} \int \wp x \cdot \exp \left[-\frac{i}{\hbar} I\left(x_{i}\right)\right]
$$

where $I\left(x_{i}\right)$ is the action 'integral' for each path $x_{i}$, leading to a wave function for each path $x_{i}$ ?

$$
\psi \sim \exp \left[-\frac{i}{\hbar} I\left(x_{i}\right)\right]
$$

If we do a rescale $\hbar=1$, then the above wave equation can lead to a Schrodinger equation,

The example given by (Lee, 2010) is that there is a Hamiltonian for which

$$
H(\phi)=\int \mathrm{d}^{3} x \cdot\left\{\frac{\left(\frac{\partial \phi}{\partial t}\right)^{2}+(\nabla \phi)^{2}+2 V(\phi)}{2}\right\}
$$

Here, $V(\phi)$ is a potential, and $\phi$ can have arbitrary values before measurement, and to a degree, $Z$ represent uncertainty in measurement. In Rindler co-ordinates, $H \rightarrow H_{R}$, in co-ordinates $\left(\eta, r, x_{2}, x_{3}\right)$ with proper time variance and co-ordinate $\eta$, then 


$$
H_{R}(\phi)=\int \mathrm{d} r \mathrm{~d} x_{\perp} a r \cdot\left\{\frac{\left(\frac{\partial \phi}{\partial r}\right)^{2}+\left(\frac{\partial \phi}{\operatorname{ar} \partial \eta}\right)^{2}\left(\nabla_{\perp} \phi\right)^{2}+2 V(\phi)}{2}\right\}
$$

Here, the $\perp$ is a plane orthogonal to the $(\eta, r)$ plane. If so then

$$
\begin{aligned}
& Z=\text { Trace }(\exp [-\beta H]) \\
& \underset{\text { transformed to }}{\longrightarrow} Z_{R}=\text { Trace }\left(\exp \left[-\beta H_{R}\right]\right)
\end{aligned}
$$

\section{Now, for the Above Situation, the Following Are Equivalent}

1) $Z_{R}$ Thermal partition function is from information loss about field beyond the Rindler Horizon

2) QFT formation is equivalent to purely information based statistical treatment suggested in this paper

3) QM emerges from information theory emerging from Rindler co-ordinate

Lee also forms a Euclidian version for the following partition function, if $I\left(x_{i}\right)$ is the Euclidian action for the scalar field in the initial frame. I.e.

$$
Z_{Q}^{E}=N_{1} \int \wp x \cdot \exp \left[-\frac{i}{\hbar} I_{E}\left(x_{i}\right)\right]
$$

There exist analytic continuation of $\tilde{t} \underset{\text { analytic continuation }}{\longrightarrow}$ it leading to

$$
\begin{aligned}
Z_{Q}^{E} & =N_{1} \int \wp x \cdot \exp \left[-\frac{i}{\hbar} I_{E}\left(x_{i}\right)\right] \underset{\text { analytic continuation }}{\longrightarrow} Z_{Q} \\
& =\text { temperature QM partition function of } Z_{Q} \text { for } \phi \text { fields }
\end{aligned}
$$

Important Claim: The following are equivalent

1) $Z_{R}$ And $Z_{Q}$ are obtained by analytic continuation from $Z_{Q}^{E}$

2) $Z_{R}$ And $Z_{Q}$ are are equivalent. 